ÉGYPTE monde arabe

\section{Égypte/Monde arabe}

$2 \mid 2005$

Les architectures constitutionnelles des régimes

politiques arabes

\title{
Turquie : la constitutionnalisation inachevée
}

Jean Marcou

\section{(2) OpenEdition}

Journals

Édition électronique

URL : https://journals.openedition.org/ema/1054

DOI : 10.4000/ema.1054

ISSN : 2090-7273

Éditeur

CEDEJ - Centre d'études et de documentation économiques juridiques et sociales

Édition imprimée

Date de publication : 31 décembre 2005

Pagination : 53-73

ISSN : 1110-5097

\section{Référence électronique}

Jean Marcou, «Turquie : la constitutionnalisation inachevée », Égypte/Monde arabe [En ligne], 2 | 2005, mis en ligne le 08 juillet 2008, consulté le 07 juillet 2022. URL : http://journals.openedition.org/ema/ 1054 ; DOI : https://doi.org/10.4000/ema.1054

Ce document a été généré automatiquement le 7 juillet 2022

Tous droits réservés 


\title{
Turquie : la constitutionnalisation inachevée
}

\author{
Jean Marcou
}

1 La Turquie est généralement considérée comme un pays à part au sein du monde musulman. Cette spécificité est le plus souvent attribuée à la laïcité qui a marqué la construction de son État-nation. Mais elle apparaît également bien dans son histoire constitutionnelle ancienne, riche et variée.

2 Il n'est pas inutile de rappeler que, très tôt, l'Empire ottoman se singularisa en générant, en dehors même de la loi religieuse, une sorte de droit public, le Kanun. Süleyman 1 1 , que les Occidentaux ont appelé « le Magnifique », est resté pour les Turcs Kanuni, c'est-à-dire, «le juriste », « le législateur ». Pourtant, c'est au moment de sa décadence que l'empire a commencé à emprunter les voies qui vont le conduire à ses premières expériences constitutionnelles. Comme l'Égypte de Méhémet Ali, l'empire se lance alors à corps perdu dans une entreprise de modernisation, s'inspirant le plus souvent de l'Occident. Mais ce n'est que sur le tard que ce mouvement prend une dimension véritablement constitutionnelle, lorsque les intellectuels se mettent à revendiquer une citoyenneté ottomane, en opérant une synthèse entre le libéralisme occidental et les traditions musulmanes.

Bien que cette première aspiration à la citoyenneté ait été submergée au début du XX⿳亠丷厂 siècle par la montée du nationalisme, le premier conflit mondial et une soif renouvelée de modernisation, la recherche d'une citoyenneté turque ne cesse pas. La république autoritaire de Mustafa Kemal, en l'absence de démocratie, prétendra promouvoir un citoyen d'un nouveau genre. Éduqué, travailleur, vertueux et pétri de valeurs progressistes, ce dernier n'est pas sans rappeler, par certains côtés, l'homme nouveau que l'urss tente de créer à la même époque. Mais le kémalisme ne se laissera jamais tenter par un totalitarisme comparable à celui des régimes qui dominent alors la scène européenne. Et surtout, en transformant le statut de la femme, il change durablement la société turque traditionnelle, tandis qu'il pérennise une série d'institutions politiques modernes, qui se transformeront par la suite. 
4 À l'issue de la Seconde Guerre mondiale, dans un contexte où les démocraties triomphent, la démocratisation est à l'ordre du jour en Turquie. Ainsi, à l'heure où le Portugal et l'Espagne sont encore des dictatures, ce pays s'essaye à la démocratie parlementaire. Mais cette tentative se révèle laborieuse. D'ouvertures politiques en coups d'État, se noue un véritable cercle vicieux de la démocratisation en Turquie. Comme déjà à la fin de l'Empire ottoman, lors des premières velléités de démocratisation de la Révolution "Jeunes-Turcs", la question constitutionnelle est au cœur du débat, mais la démocratie et l'État de droit tardent à se concrétiser.

5 Antériorité et actualité, tels sont bien les caractères du processus constitutionnel en Turquie et tels seront les axes essentiels de notre réflexion. L'observation des racines du constitutionnalisme turc surprend par l'ancienneté de celles-ci, qui peut rivaliser avec celles du constitutionnalisme de bien des États européens, du sud notamment. Pour autant, la constitutionnalisation du pouvoir ne paraît pas véritablement achevée, au moment même où ce pays frappe à la porte de l'Union européenne avec de plus en plus d'insistance.

\section{L'antériorité de l'expérience constitutionnelle turque et ses héritages ottoman et kémaliste}

$6 \quad$ L'histoire politique et constitutionnelle de la Turquie contemporaine ne commence pas avec la fondation de la République en 1923, mais dans les dernières décennies de l'Empire ottoman. Au cours du xixe siècle, en effet, alors que cet empire essaye désespérément d'enrayer son déclin, vont se développer une modernisation ambitieuse et un débat intellectuellement riche sur la forme de l'État, l'expression de la souveraineté, les équilibres institutionnels, les questions de citoyenneté et de nationalité. Les idées et les expériences constitutionnelles de cette époque vont fortement contribuer en réalité à l'avènement de l'État-nation turc moderne qui, en dépit de son autoritarisme initial, saura ménager une place particulière au constitutionnalisme.

\section{Modernisation, réformisme et constitutionnalisation dans l'Empire ottoman en déclin}

7 Les Lumières et les idées des grandes révolutions libérales n'ont touché que tardivement la Méditerranée orientale. Les premières réformes politiques engagées dans l'Empire ottoman visaient surtout à moderniser ce dernier en utilisant des recettes techniques d'inspiration occidentale. Elles ont donc émané, au départ, d'une élite gouvernante diplomatico-militaire et ont commencé à prendre corps sous le règne des sultans réformateurs Sélim III (1789-1807) et Mahmud II (1808-1839). Déçus par le caractère hâtif et souvent formel des mesures prises, les observateurs occidentaux de l'époque eurent tendance à ne voir, en ces despotes éclairés, que de pâles imitateurs de Pierre le Grand. Pourtant, il ne faut pas négliger le fait que le long règne de Mahmud II, notamment, conduisit à une remise en cause de certains des fondements de l'empire, en permettant, en particulier, l'abolition de la féodalité ottomane (avec la suppression des timar), la fondation d'une armée moderne (avec l'élimination du corps des Janissaires), la création de ministères et d'administrations centrales, le renforcement de la 
centralisation, le développement d'un enseignement des langues étrangères et de nouvelles disciplines, destiné à promouvoir la formation de cadres compétents.

Toutefois, les évolutions ultérieures allaient prendre un tour beaucoup plus radical par la suite. En 1839, en effet, au début du règne du fils de Mahmud II, Abdul Mejid I - avec le fameux noble rescrit de Gülhane (Hatt-i-serif), qui fut confirmé en 1856 par un second rescrit (Hatt-i-humayun) - un fort mouvement de modernisation et d'occidentalisation, mieux connu sous le nom de "période des Tanzimat ", commença à affecter l'empire, marquant les règnes de trois sultans et s'étalant sur près de quatre décennies. Bien que certains aient cru devoir faire, un peu vite, de ces textes les éléments d'une Magna Carta a la turca, ce mouvement doit être analysé avec prudence. D'une part, sa dimension politique resta limitée, les réformes étant techniques et souvent très en décalage avec les réalités de la société ottomane de l'époque. D'autre part, le contexte international et, dans ce cadre, les pressions exercées par les Occidentaux furent aussi largement à l'origine de ces textes. Ce fut en partie pour prouver aux puissances occidentales que la Sublime Porte, à l'instar du pacha d'Égypte, Méhémet Ali, pouvait promouvoir un régime libéral, que Mustafa Resid pacha, l'un des principaux architectes des réformes des Tanzimat rédigea le Hatt-i-Serif. Ce fut à nouveau pour donner des gages aux puissances européennes, à la veille du Traité de Paris, et pouvoir entrer dans le concert européen, que le sultan promulgua le Hatt-i-humayun, qui développait les garanties des non-musulmans de l'empire, en affirmant de manière nette l'égalité des sujets ottomans quelle que soit leur religion. Il reste que les Tanzimat, en permettant de multiples transformations juridiques, administratives, militaires, fiscales ou éducatives, approfondirent et élargirent la réflexion sur ce que devait être l'avenir de l'empire. De ce fait, ils rendirent inévitable la revendication constitutionnelle.

En effet, au terme de ce cycle de modernisation, survint enfin la promulgation de la première et de la seule constitution ottomane, la Constitution de 1876. Loin d'apparaître à l'époque comme la consécration d'un mouvement de fond, ce texte fut même considéré par beaucoup comme une manœuvre de la Sublime Porte pour conjurer de nouvelles ingérences occidentales, puisqu'il fut proclamé, de façon inattendue, à Istanbul au début d'une conférence internationale à laquelle l'empire avait dû consentir sous la pression des grandes puissances. Pour autant, comme le dit Bernard Lewis, il serait inexact d'en conclure que la promulgation de cette constitution et la réunion du premier Parlement ottoman qu'elle provoqua, ne furent que «des subterfuges diplomatiques destinés à abuser les étrangers sans rien changer dans le pays » (1988, p. 149). En fait, non seulement l'édiction de cette constitution avait été précédée par le mouvement réformateur que nous venons d'évoquer, mais il faisait suite à une crise politique interne profonde qui, au cours de l'année 1876, avait conduit à la déposition du sultan Abdül Aziz et à l'organisation laborieuse de sa succession dans un contexte où, de surcroît, la revendication constitutionnelle avait été présente en permanence.

10 Fortement inspirée par la Constitution belge de 1831, parce que celle-ci organisait une monarchie et qu'elle était écrite dans une langue (le français) bien maîtrisée par les élites ottomanes, la nouvelle Constitution ne prétendait pas émaner de la souveraineté du peuple mais de la volonté du sultan. Confiant le pouvoir exécutif à ce dernier resté calife et à un Premier ministre (qui reprendra par la suite le nom de "Grand vizir »), elle instaurait, néanmoins, un pouvoir législatif bicaméral et ébauchait des mécanismes 
de vie parlementaire, ce qui permit la tenue d'élections et le déroulement de premiers travaux d'assemblée.

11 Les passages les plus intéressants de ce texte de 119 articles concernent néanmoins le statut de l'État ottoman et la création d'une citoyenneté ottomane. Compte tenu du contexte de la promulgation de la Constitution auquel nous faisions précédemment allusion, on peut penser que ces dispositions n'étaient pas exemptes de préoccupations tactiques et diplomatiques, qui visaient à protéger l'empire des ingérences extérieures au nom même de principes venant de l'Occident. Mais elles reflétaient sans doute aussi une réflexion politique plus fondamentale, soucieuse du développement d'une réelle citoyenneté et inspirée par le modèle de l'État-nation libéral. Ainsi, le premier article de la Constitution prétendait garantir l'intégrité territoriale de l'Empire ottoman, en le définissant comme « un tout indivisible dont aucune partie » ne pouvait « être détachée pour quelque motif que ce soit ». À cela, l'article 8 ajoutait la reconnaissance d'une sorte de nationalité ottomane («Tous les sujets de l'empire sont indistinctement appelés Ottomans, quelle que soit la religion qu'ils professent »). La connaissance du turc était néanmoins exigée pour pouvoir siéger au Parlement (cf. art. 68). Enfin, au terme de l'article 11, l'islam était religion d'état, mais la liberté de culte était garantie et tous les sujets ottomans devenaient des citoyens jouissant des mêmes droits et libertés (égalité devant la loi, liberté individuelle, droit de propriété, sûreté, droit d'association, liberté de la presse, liberté de l'enseignement, égalité d'accès aux fonctions publiques...).

Bien que de premières élections législatives aient pu se tenir et permettre à des parlementaires de siéger pendant quelques mois, le rayonnement de ce premier texte constitutionnel demeura limité. En effet, après avoir destitué en 1877 le Grand vizir, Midhat Pasa, qui avait été le principal rédacteur de la Constitution, le sultan Abdül Hamid, en 1878, exaspéré par la tournure que prenaient ces débats suite aux graves revers subis par l'empire face aux Russes, décida de renvoyer le Parlement. Ce dernier ne devait plus se réunir pendant trente ans.

\section{L'approfondissement de l'expérience constitutionnelle ottomane à la fin de l'Empire}

13 La première expérience constitutionnelle ottomane de 1876-78 fut largement ignorée par l'analyse politique et constitutionnelle occidentale. Très dépendante des contingences internationales, elle paraissait consacrer l'échec de plusieurs décennies réformatrices. Les Tanzimat n'avaient fait qu'imiter l'Occident, ils n'étaient pas parvenus à promouvoir une transformation politique et sociale de l'empire en profondeur. Pourtant, une telle opinion mésestimait, en réalité, la portée du réformisme ottoman. Ces trains de mesures, d'essence plutôt technique, avaient de fait progressivement remis en cause les rouages traditionnels de la société et de l'État ottomans, provoquant l'apparition d'une réflexion politique critique, dont témoigne bien l'épopée du mouvement des "Jeunes-Ottomans " auquel succédera, après la suspension de la Constitution de 1876, le mouvement "Jeunes-Turcs ». C'est, en effet, au sein même de ces formations politiques ottomanes naissantes que les fondements de la culture constitutionnelle et politique turque vinrent à maturité.

Les «Jeunes-Ottomans » furent directement à l'origine de la première revendication constitutionnelle dans l'empire. Leurs idées s'imposèrent au moment où les Tanzimat 
commencèrent à montrer leurs limites. Issu essentiellement des nouveaux milieux littéraires et artistiques admirateurs de la culture occidentale, ce mouvement, s'inspirant du modèle Carbonari, se constitua à partir de 1867 en société secrète. Animé principalement par Ziya Pasa, le prince égyptien Mustafa Fazil et surtout Namik Kemal, il allait se lancer dans une critique acerbe de la monarchie ottomane déclinante, qui s'essayait péniblement à la réforme depuis plusieurs décennies (Dumont, 1989, p. 464 à 468). Les « Jeunes-Ottomans » mirent ainsi le doigt sur les contradictions des Tanzimat qu'ils analysèrent comme une occidentalisation administrative aux effets pervers. En copiant les formes occidentales, en sécularisant le droit et l'enseignement, en entamant des institutions religieuses comme la sharîa et l'appareil éducatif des oulémas, ce volontarisme réformateur avait fait disparaître les instances qui équilibraient le pouvoir du souverain et avait, de fait, désorganisé le système ottoman. Au moment même où l'on parlait de réforme et de modernisation, jamais l'État et le sultan n'avaient été aussi puissants. Face à ce constat, les «Jeunes-Ottomans » réclamaient l'avènement d'un gouvernement constitutionnel et d'une véritable citoyenneté. Ils devinrent ainsi l'opposition libérale au despotisme réformateur du sultan. C'est donc véritablement avec eux que les idées de la Révolution française touchèrent l'empire en profondeur, mais principalement pour servir de support à une critique ottomane de la modernisation conduite jusqu'alors. L'originalité profonde de ce courant de pensée est, en effet, d'avoir tenté d'associer un idéal constitutionnaliste d'origine occidentale à la revendication du maintien des droits et traditions islamiques (Lewis, 1993, p. 83). Pour Namik Kemal, notamment, l'existence d'un gouvernement constitutionnel responsable devant une assemblée élue peut trouver sa justification dans le Coran et la loi islamique. Ces penseurs favorisèrent ainsi l'adaptation de concepts constitutionnels et politiques libéraux à une société orientale, comme par exemple ceux de "Patrie " (Vatan) ou de «Liberté » (Hürriyet), leurs deux mots d'ordre. Un tel effort de théorisation peut être considéré comme le prélude intellectuel et politique qui engendra la première expérience constitutionnelle ottomane. Mais l'échec de cette expérience fut aussi l'échec des «Jeunes-Ottomans ». En 1876, après la déposition du sultan Abdül Aziz, alors qu'était arrivé au pouvoir un sultan favorable au constitutionnalisme (Murat v), leurs projets furent contrariés par un contexte international difficile et par l'incapacité de ce sultan à exercer le pouvoir. Son successeur, Abdül Hamid, après avoir accepté dans un premier temps la monarchie constitutionnelle qu'on lui proposait, en vint, sous la pression de la conjoncture, à un tout autre dessein: provoquer le sursaut de l'empire, en rétablissant un régime autoritaire plus respectueux des traditions islamiques de l'empire. Les «JeunesOttomans » furent les premières victimes de cette réaction hamidienne et disparurent de la scène politique. Pourtant, ce retournement ne fit pas disparaitre l'idéal constitutionnel car la remise en application de la Constitution de 1876, qui n'avait été que suspendue, devint le mot d'ordre de nouveaux opposants.

C'est en effet un autre mouvement, celui des "Jeunes-Turcs ", né dans la clandestinité en 1889, l'année du centenaire de la Révolution française, qui allait faire renaître et amplifier la revendication constitutionnelle sous la dictature hamidienne. Ces nouveaux activistes avaient des idées sensiblement différentes de celles de leurs prédécesseurs. Pour faire évoluer l'empire, il fallait, selon eux, le transformer par la science, l'éducation, le droit et le doter d'un régime politique moderne et rationnel assis sur une constitution. Influencés comme les « Jeunes-Ottomans », par les Carbonari 
italiens pour leur organisation, c'était désormais plutôt dans le rationalisme, le positivisme et le scientisme qu'ils trouvaient l'essentiel de leur inspiration idéologique.

Il faut voir en outre que la conjoncture internationale confortait leur constitutionnalisme enthousiaste. Le Japon constitutionnalisé n'avait-il pas eu raison de la Russie tsariste qui avait dû se doter d'une constitution après la révolution de 1905, ce à quoi l'Empire perse avait dû lui aussi se résoudre en 1906 (Badie, 1986, p. 165 et s.) ? Ainsi, pour cette nouvelle génération de modernisateurs, seul le remède constitutionnel pourrait guérir «l'homme malade ».

Toutefois, au fur et à mesure que la revendication constitutionnelle s'intensifiait, elle mettait à jour la complexité inhérente au projet de constitutionnalisation d'un tel empire. Les textes fondateurs des Tanzimat et le texte de 1876 s'étaient déjà fortement préoccupés du problème du statut juridique des sujets ottomans. Leur universalisme humaniste proclamé n'était pas sans ambiguïté appliqué aux réalités d'une société ottomane, compartimentée en "millet», ces entités communautaires nationaloreligieuses dans lequel les Ottomans vivaient leur vie civile, et parfois l'essentiel de leur vie sociale.

18 La revendication d'une citoyenneté ottomane - qui, nous l'avons vu était en partie satisfaite par la Constitution de 1876 et sur laquelle les peuples de l'empire s'accordaient - rejoignait la question de la forme que devrait avoir le futur État ottoman constitutionnalisé. Fallait-il promouvoir un État nation unitaire turc ou lui préférer une fédération plus attentive aux droits des différents peuples et minorités de l'empire? Ce dilemme scinda profondément le mouvement «Jeunes-Turcs» en 1902, lors de son congrès de Paris, en deux tendances : celle du " comité Union et Progrès » conduite par Ahmet Riza, celle de la «Ligue pour l'initiative privée et la décentralisation » fondée par le prince Sabaheddin. Cette scission entre nationalistes turcs et libéraux ottomans devait avoir, par la suite, un retentissement dépassant largement le contexte politique de l'époque. Elle fut, en effet, à l'origine d'une dichotomie idéologique essentielle pour la compréhension de la structuration du système partisan turc et opposant un courant jacobin, laïque, progressiste, étatiste (" comité Union et Progrès », kémalisme, sociaux-démocrates populistes) à un courant libéral moins centralisateur et plus sensible à la tradition religieuse (Ligue pour l'initiative privée et la décentralisation, Parti démocrate, Partis contemporain de centre droit).

19 Pourtant, ni les débats idéologiques ni la mobilisation citoyenne ne furent déterminants dans le déclenchement de la révolution "Jeunes-Turcs» en juillet 1908, bien que cet événement ait laissé le souvenir de la fraternisation spectaculaire, dans les grandes villes, des différents peuples de l'empire célébrant la fin du despotisme hamidien. Cette révolution fut, au départ, un mouvement patriotique d'officiers turcs musulmans qui voulaient sauver l'empire. En ce sens, il faut y voir un moment important dans la marche vers l'émergence d'un État nation turc qui mit également en relief la place qui pouvait être celle de l'armée dans le changement politique et social. Cette dimension nationaliste n'enlève rien à la richesse de l'événement car le rétablissement de la Constitution de 1876 qu'il permit déboucha sur une seconde période constitutionnelle ottomane. Entre 1908 et 1913, celle-ci fut aussi la première expérience réelle de monarchie parlementaire dans l'empire. La société ottomane découvrit les joutes électorales, la liberté de la presse, le droit de réunion, les grèves et 
même les revendications féministes. On assista, en outre, à un développement sans précédent de l'édition et des débats politiques, religieux et philosophiques.

Cependant, cette seconde période constitutionnelle acheva de démontrer le caractère illusoire de la réforme ottomane. Il est sûr que, là encore, la conjoncture internationale défavorable (guerre de Tripolitaine, guerres balkaniques...) a sa part de responsabilité, mais l'échec de la constitutionnalisation de l'empire tentée alors a aussi des raisons tout à fait indigènes que l'on peut exposer comme suit. En premier lieu, cette période devait mettre clairement en relief l'impossibilité de faire d'une citoyenneté ottomane fragile une véritable nationalité ottomane. En effet, l'emprise des unionistes sur le gouvernement favorisa le développement d'un phénomène nationaliste de turquisation des citoyens de l'empire, largement justifiée aux yeux de l'opinion dominante par les menaces sécessionnistes découlant de la montée des nationalités dans les Balkans. En second lieu, le débat politique dans ce régime parlementaire naissant, et en particulier l'opposition entre libéraux et unionistes, loin de déboucher sur la formation du système constitutionnel reposant sur le bipartisme et l'alternance que certains appelaient de leurs vœux (Fesh, 1909, p. 61), dégénérèrent très rapidement en conflits ouverts : émeutes, interventions militaires, assassinats politiques, dissolutions du parlement, fraudes électorales... Ainsi dès 1913, le nouveau régime sombra dans une sorte d'oligarchie militaire dirigée par le triumvirat unioniste d'Enver, Talat et Cemal, s'éloignant de plus en plus du rêve d'un État ottoman constitutionnel, parlementaire et décentralisé. Ce triumvirat et ses dérives devaient conduire l'empire à sa perte en le faisant entrer en guerre aux côtés de l'Allemagne et de l'Autriche-Hongrie.

\section{Les ambiguïtés du constitutionnalisme kémaliste}

Le déploiement incantatoire de la Constitution, du Parlement, du Parti et des élections ne saurait cacher le fait essentiel que la république fut établie par un soldat de métier à la tête d'une armée victorieuse qui se maintint au pouvoir, au début tout au moins, par un mélange d'autorité paternelle et de puissance militaire. (Lewis, 1988, p. 324)

Cette remarque de Bernard Lewis montre bien que l'épopée kémaliste fut plus autoritaire qu'à proprement parler constitutionnaliste. Pour autant, l'architecture constitutionnelle du kémalisme ne fut pas qu'un attribut de façade. Héros de la guerre d'indépendance, Mustafa Kemal aurait pu se contenter d'établir une dictature et jouir facilement de son succès jusqu'à la fin de ses jours. Il choisit de mettre un terme à sa carrière de général victorieux pour devenir le président civil d'une république, qui ambitionnait de transformer profondément le peuple et la société que la guerre d'indépendance venait de sauver. Pour cet ancien militant « Jeunes-Turcs », admirateur de la Révolution française, né à Salonique, la ville d'où était partie la révolution constitutionnelle de 1908 et où s'étaient tenus, parfois plus qu'à Istanbul, les grands débats d'idées de la fin de l'empire, le concept de constitution ne pouvait qu'avoir une valeur éminente. Le régime kémaliste ne fut, toutefois, ni une démocratie libérale ni un État de droit et il demeurera sans doute éternellement une énigme à son sujet quant à sa nature et ses objectifs ultimes. Était-il une phase transitoire de dictature destinée à conforter les réformes entreprises et à permettre, à terme, l'établissement d'une démocratie véritable ? Était-il un régime sui generis destiné à promouvoir, avant tout, une modernisation de la société turque qui ne devait pas nécessairement déboucher sur un système de type libéral ? L'autoritarisme, les dérapages idéologiques du kémalisme dans les années trente et le caractère global des réformes entreprises font pencher 
pour la seconde hypothèse et amènent même à s'interroger sur l'existence d'une dérive de type totalitaire, dans laquelle le régime ne sombra pourtant pas. En revanche, tant le caractère très occidentaliste du discours officiel de l'époque sur la nécessité de «civiliser» le peuple turc que les deux tentatives avortées d'expériences pluralistes montrent que la première hypothèse est toujours restée à l'ordre du jour. En tout état de cause, même si le modèle occidental était demeuré influent dans la république kémaliste, il faut bien voir que la période au cours de laquelle elle se développa (l'entredeux-guerres), fut marquée par la crise des démocraties libérales d'Europe occidentale et par la montée en force de nouveaux systèmes nationalistes, fascistes, communistes... Quoi qu'il en soit, le constitutionnalisme de Mustafa Kemal n'était sûrement pas d'essence libérale mais fortement empreint d'un progressisme que n'auraient pas renié beaucoup de républicains français de la fin du xixe siècle. Par la création de nouvelles institutions et d'idéaux nationaux non dénués d'une dimension universaliste, une constitution devait contribuer, dans son esprit, à transformer la société pour permettre au peuple d'assumer son destin. Avec le progrès et l'instruction publique, ce cadre idéal et un peu formel deviendrait un jour une réalité sociale. En ce sens, loin de n'être qu'un alibi, l'architecture constitutionnelle kémaliste doit être considérée comme un caractère essentiel de la nouvelle république turque. À bien des égards, une constitution permettait de synthétiser les idéaux du régime en affirmant l'existence d'une nouvelle structure étatique nationale où des réformes radicales étaient appelées à se dérouler et à façonner le citoyen turc de demain (Fuad Basgil,1939). En fait, ce constitutionnalisme modernisateur kémaliste renouait un peu avec l'idéal des réformateurs ottomans, en particulier avec celui des «Jeunes-Turcs». Une élite réformatrice dominée par l'armée, qui avait déjà été à l'origine de la Révolution de 1908, tentait, en fondant un nouvel État, de créer les conditions nécessaires à l'émergence d'une citoyenneté moderne. Avec la référence à la souveraineté de la nation, la construction constitutionnelle kémaliste permettait de lier la cause nationale et la cause citoyenne. Cette invocation constitutionnelle n'était pas seulement destinée à affirmer sur la scène internationale l'existence d'une Turquie nouvelle, elle voulait aussi, à l'origine, légitimer le gouvernement nationaliste face au gouvernement ottoman qui avant 1923 était encore le gouvernement officiel de la Turquie. C'est ce qui explique que même le gouvernement provisoire d'Ankara, pendant la guerre d'indépendance, éprouva le besoin d'inscrire son existence et son action dans un cadre constitutionnel - celui de la Constitution de 1921 - et que, dès 1924, la jeune république turque fut dotée d'un texte fondamental. Pour prendre le pouvoir, Mustafa Kemal aurait pu se contenter de la force militaire et de ses victoires. Il voulut en réalité donner à la destitution du sultan l'aspect d'une révolution démocratique. Dès lors, comme l'expliquait en 2001 le professeur Mümtaz Soysal, lors de la conférence internationale de droit constitutionnel d'Ankara (Uluslararasi anayasa hukuku kurultayi, 2001, p. 85), ce n'est pas un hasard si la Constitution de 1924 instaurait un régime d'assemblée proche du régime conventionnel français jacobin de 1793/94. Même si ce n'était qu'une fiction, elle voulait en fait donner le pouvoir à la Grande Assemblée nationale de Turquie (GANT), qui avait incarné la résistance de la nation turque pendant la guerre d'indépendance et qui serait, demain, l'émanation d'un peuple instruit et travailleur capable de prendre en main ses destinées. Ainsi, il s'agissait d'une constitution simple, reposant sur l'idée de gouvernement du peuple. Mustafa Kemal avait défini dès 1921 l'esprit du régime politique qu'il voulait créer en ces termes : 
$\mathrm{Si}$ nous devions définir sociologiquement notre système politique, nous l'appellerions le gouvernement du peuple... Nous sommes des travailleurs, des pauvres, qui s'efforcent de sauver leur vie et l'indépendance.... Est-ce notre faute si nous ne ressemblons pas à la démocratie, si nous ne ressemblons pas au socialisme, si nous ne ressemblons à rien? Messieurs, nous devrions être fiers de défier toute comparaison! Parce que, messieurs, c'est à nous-mêmes que nous ressemblons ! (dans Lewis, 1988)

22 Au-delà des déclarations d'intentions et même si le pouvoir devait être, en réalité, exercé par une élite, cette constitution se voulait surtout, dans sa simplicité, porteuse des potentialités qui devaient lui permettre de générer et d'encadrer les évolutions futures. Pourtant, elle n'a qu'en partie fait la preuve qu'elle était apte à tenir ce rôle, car si elle a bien été le cadre d'une première expérience de démocratie pluraliste dans les années cinquante, elle n'y a pas survécu. La place qui est la sienne dans le constitutionnalisme turc n'est pas négligeable pour autant. Incarnant l'esprit républicain populiste qui est à la base de la fondation de l'État nation turc contemporain, elle reste un texte fondamental de l'histoire constitutionnelle turque dont nous voulons, maintenant, évoquer les principaux apports.

\section{La Constitution de 1924et l'héritage constitutionnel kémaliste}

Le premier apport de la Constitution kémaliste de 1924 concerne, à notre avis, la souveraineté nationale et son exercice. Certes, la période kémaliste ne fut pas démocratique et déboucha sur l'instauration d'un régime de parti unique mais, en faisant du peuple ou plus exactement de la nation, même formellement, le titulaire de la souveraineté, cette constitution a consacré l'avènement définitif d'une nouvelle conception laïcisante et non-autocratique de la souveraineté. Il est vrai que ce processus avait été lancé pendant la seconde expérience constitutionnelle ottomane. C'est en effet en 1909, lors de la révision constitutionnelle suivant les émeutes qui conduisirent à la déposition d'Abdül Hamid, que fut évoquée pour la première fois l'idée de la souveraineté du peuple. Mais c'est surtout dans le contexte de la guerre d'indépendance que l'idée progressa. À cet égard, la Constitution provisoire de 1921 marqua un tournant puisqu'elle s'ouvrait par cette déclaration définitive: «La souveraineté appartient sans réserve ni condition à la nation; le système d'administration repose sur le principe que le peuple dirige personnellement et effectivement sa propre destinée. » Dans son article 3, la Constitution républicaine de 1924 consacra l'affirmation de la souveraineté nationale. Toutefois, il est important d'observer que cette affirmation ne s'accompagnait pas de la reconnaissance d'une véritable séparation des pouvoirs. Ainsi, la GANT était placée au cœur du système et même le pouvoir exécutif relevait formellement de l'instance qui était sensée incarner la nation. Seul le pouvoir judiciaire se voyait reconnaître une certaine autonomie, tant dans le premier chapitre de la Constitution que dans le quatrième chapitre qui lui était spécialement consacré. Toutefois, cette unicité dans l'exercice de la souveraineté allait marquer les premiers développements de l'histoire constitutionnelle turque et induire des phénomènes pervers dans le contexte de la démocratisation qui interviendra postérieurement.

24 Le second apport de la Constitution concerne le legs d'une morphologie institutionnelle libérale au système politique turc. En effet, en dépit de l'absence de démocratie, la période kémaliste a permis d'ancrer dans la société politique turque des formes 
institutionnelles qui seront par la suite à la base de la démocratisation du pouvoir : le président de la République, la Grande Assemblée nationale, le Premier ministre et son gouvernement, le pouvoir judiciaire.... En dépit de l'absence de séparation des pouvoirs que nous soulignions précédemment, les différents pouvoirs sont successivement décrits par le texte constitutionnel. À bien des égards, cet apport fait penser à celui des dispositions institutionnelles de la Constitution portugaise de 1976. En inscrivant dans sa loi fondamentale l'existence d'institutions telles qu'un président, un Premier ministre, un Parlement, la jeune démocratie portugaise, qui hésitait alors entre libéralisme et socialisme, jetait les bases d'une parlementarisation du régime qui fut consacrée par la révision constitutionnelle de 1982. En Turquie, cette morphologie institutionnelle initiale permettra une parlementarisation progressive du système kémaliste, après la Seconde Guerre mondiale, et le développement d'une première expérience de démocratie parlementaire entre 1950 et 1960.

Le dernier apport de la Constitution de 1924 au constitutionnalisme turc concerne une série de concepts qui, même s'ils n'ont pas toujours survécu en l'état par la suite, ont néanmoins durablement marqués les structures politiques et constitutionnelles turques car ils concernent, finalement, la forme du nouvel État nation et le statut moderne du citoyen turc qui se révèle, d'ailleurs, être aussi une citoyenne. La version révisée de la Constitution fondait l'État turc, dans son article 2, sur six principes (républicanisme, nationalisme, populisme, étatisme, laïcisme et réformisme) qui étaient également ceux du parti unique, le Parti républicain du peuple (les six flèches du kémalisme). En réalité, trois de ces principes ont durablement marqué l'État turc: le laïcisme, le républicanisme et le nationalisme.

Le laïcisme peut être considéré comme le premier d'entre eux car en dépit des évolutions postérieures, la laïcité s'est maintenue et reste l'une des originalités profondes de la Turquie contemporaine, l'un des très rares États, avec la France, à l'avoir inscrite dans son texte constitutionnel (Marcou, 2000). L'affirmation constitutionnelle de la laïcité turque, pourtant, n'émanera pas directement de la Constitution de 1924. En effet, dans son article 2, celle-ci faisait initialement de l'islam une religion d'État. Ce n'est que par une révision du 10 avril 1928 que cette disposition sera modifiée. En réalité, l'affirmation du principe de laïcité s'étalera sur plus de dix ans après la fondation de la République, même si l'idée de séparer État et religion, ou plus exactement d'encadrer strictement, selon la conception turque, l'exercice de la religion était déjà en germe dans certains textes adoptés par la GANT au cours de la guerre d'Indépendance. La consécration du principe de laïcité découlera, en fait, d'amendements successifs à la Constitution et de trains de mesures législatives issus du réformisme radical de l'époque, comme par exemple, la série de lois de 1924, 1925 et 1926 qui mettront fin au califat, supprimeront les congrégations religieuses, unifieront l'enseignement et instaureront le mariage civil. Au bout du compte, force est de constater que la Turquie a inscrit la laïcité dans son ordre constitutionnel avant la France, qui ne le fera qu'en 1946.

Le républicanisme est un legs également à prendre en compte. La république incarne en Turquie d'abord le caractère intangible de la forme nouvelle de gouvernement face à toute idée de retour à la monarchie et à l'ordre social ancien, principe qui fut d'ailleurs affirmé avant même la Constitution de 1924, notamment par la loi n 431 du 3 mars 1924 qui non seulement supprimait le califat, mais ordonnait également le bannissement immédiat de tous les membres de la famille royale. Toutefois, la 
république renvoie aussi, dans ce pays, à la citoyenneté et on observe que la Constitution de 1924 comporte une déclaration de droits importante, dont certains développements ne sont pas sans rappeler la déclaration française de 1789. Il est évident que ces dispositions ne suffiront pas à faire de la République kémaliste un État de droit, mais ce texte a initié, malgré tout, un changement important du statut de la personne en Turquie - en particulier en ce qui concerne l'égalité entre hommes et femmes, avec notamment la reconnaissance du droit de vote aux femmes, après une révision constitutionnelle datant de 1934. Il n'en reste pas moins que le concept de république reste, malgré tout, ambigu car il évoque en réalité autant la souveraineté nationale et le projet d'instauration progressive d'une citoyenneté démocratique, que la nécessaire autorité de l'État face à la société civile et aux traditions religieuses (Marcou, Üstel, Vardar, 2000).

De fait, il n'est pas étonnant que le nationalisme soit le dernier trait marquant de ce constitutionnalisme turc naissant. Associé aux deux principes précédemment exposés, il a été pratiqué avec constance pour légitimer l'indivisibilité de l'État turc contemporain face aux revendications identitaires de toutes sortes. Sur le plan international, ce nationalisme n'est ni agressif ni irrédentiste. Au contraire, il rejette toute idée de pan-turquisme ou de panislamisme et servira de fondement à une politique étrangère kémaliste traditionnellement neutraliste et isolationniste. Sur le plan interne, ce nationalisme évoque, bien sûr, la souveraineté nationale puissamment affirmée, nous l'avons vu, dans l'ordre constitutionnel. Mais derrière l'unité de la nation se profile aussi l'unité de l'État sur laquelle les Turcs resteront particulièrement sourcilleux. Les Kurdes l'apprendront très vite à leurs dépens. Le régime kémaliste, hostile à toute décentralisation prononcée, reprendra, pour l'essentiel, l'organisation administrative unitaire voisine du système départemental français, issu de la fameuse loi de 1871 sur les conseils généraux, que lui avait léguée la seconde période de constitutionnelle de l'Empire ottoman (loi ottomane de 1913) et qui est toujours en vigueur aujourd'hui.

\section{La fin des illusions : vers un constitutionnalisme réel permettant le développement de la démocratie et de l'État de droit}

Nous venons de voir que l'antériorité de l'expérience constitutionnelle turque est indiscutable. C'est au cours des dernières décennies de l'empire et de la période kémaliste que se façonne la culture constitutionnelle turque autour de grands débats de fond: rôle des élites et de l'armée dans les entreprises de réforme, instauration d'une citoyenneté et d'une démocratie véritables, dilemme entre le maintien de traditions religieuses ou identitaires et le développement d'un réformisme d'inspiration occidentale. Toutefois, le constat de cette antériorité est ambigu car, si l'invocation constitutionnelle a été un phénomène permanent de l'empire puis de la république depuis le milieu du XIx siècle, les expériences de constitutionnalisation véritable sont finalement restées marginales. Ce qui frappe le plus dans l'examen de ces phénomènes, c'est qu'ils ont été moins provoqués par une revendication sociale de limitation et de contrôle de l'exercice du pouvoir, que motivés par la volonté d'accéder à une sorte de modernité politique. Ainsi, plus qu'un phénomène de constitutionnalisation du pouvoir, la République kémaliste est l'aboutissement d'un processus de modernisation 
de l'État et de la société. Pourtant, elle n'a pas enterré le mouvement constitutionnel turc car, en dépit de son caractère autoritaire, elle a implanté dans la structure politique turque des principes et des institutions qui ont été amenés à s'épanouir postérieurement. À l'issue de la mort d'Atatürk et de la Seconde Guerre mondiale, dans un contexte nouveau, la question constitutionnelle allait ainsi connaître de nouveaux développements.

\section{L'expérience de démocratie parlementaire dans le cadre de la Constitution kémaliste de 1924}

En Turquie, le passage à la démocratie ne fut pas au départ la conséquence d'un mouvement social de fond ou d'une rupture politique importante. Conformément à ce que nous avons pu observer jusqu'à présent, cette mutation devait d'abord être provoquée par les élites gouvernantes pour des raisons très pragmatiques de politique internationale (faciliter l'insertion de la Turquie dans le bloc occidental face à la menace soviétique) et de politique intérieure (donner un nouveau souffle au régime). Elle ne se traduisit ainsi par aucune réforme constitutionnelle, mais fut la conséquence d'un enchaînement de faits et de décisions qui générèrent, finalement, un changement de fond. Deux événements furent à cet égard déterminants : le discours du 1er novembre 1945 à la GANT, où Ismet Inönü (le successeur d'Atatürk) souhaita la création d'un parti d'opposition, et l'instauration du suffrage universel direct en 1946 dans un pays qui votait depuis l'Empire ottoman au suffrage indirect. Ces événements débouchèrent d'une part sur la fondation, dès 1946, d'un Parti démocrate par des dissidents du parti unique kémaliste (Parti républicain du peuple, Cumhuriyet Halk Partisi, снР) qui revendiquaient moins d'étatisme et plus de démocratie et, d'autre part, sur la victoire sans appel de ce nouveau parti aux élections générales de mai 1950. Après vingt-sept ans de règne sans partage, le cHP dut accepter de céder le pouvoir à un parti d'opposition: Celal Bayar fut élu par la nouvelle Assemblée à la présidence de la République et Adnan Menderes devint Premier ministre. La Turquie avait vécu, en l'occurrence, une rupture comparable par son ampleur à celles de 1876, de 1908 et de 1923. Même si cet événement fut initié, comme nous le disions, par les élites au pouvoir dont les desseins étaient loin d'être exclusivement idéalistes, on ne peut s'empêcher de penser qu'il illustrait l'accession de ce pays à une certaine maturité politique. Car il montrait que le gouvernement au pouvoir avait non seulement décidé d'organiser des élections libres, mais qu'il avait aussi accepté de se soumettre à leur verdict. En réalité, les élections de 1950, en faisant entrer la pratique de l'alternance dans le système politique turc contemporain, ont constitué un pas important vers la constitutionnalisation du pouvoir.

31 Pour autant, les événements qui devaient se dérouler dans les années cinquante allaient montrer que la Turquie avait du mal à dominer les conséquences de ces mutations fondamentales. En effet, le Parti démocrate exerça le pouvoir sans partage pendant une dizaine d'années. On a surtout retenu de cette époque les réformes qui conduisirent à la remise en cause des aspects les plus saillants et les plus militants de la laïcité kémaliste. En fait, le gouvernement de Menderes toucha peu aux fondements essentiels de la laïcité turque. Il s'employa surtout à tempérer l'activisme laïciste des décennies précédentes et à promouvoir une désétatisation sensible pour accompagner une politique économique d'inspiration libérale. 

qui se révéla alors. Les avatars de la décennie démocrate contribuèrent, en fait, à mettre en lumière l'illusion constitutionnelle dans laquelle vivait la Turquie, en montrant d'une part que la Constitution de 1924, restée en vigueur, s'avérait incapable d'assurer le fonctionnement du régime représentatif ou de garantir efficacement les droits fondamentaux et, d'autre part, que les acteurs politiques étaient loin d'accepter le consensus minimum nécessaire au fonctionnement d'un système politique reposant sur l'alternance. En effet, les "Républicains ", tout en consentant à l'existence d'un parti d'opposition, avaient tout fait pour l'empêcher d'accéder au pouvoir. Quant aux «Démocrates ", une fois au gouvernement, ils eurent souvent le même comportement de parti unique et appliquèrent à l'opposition républicaine, ou à la presse, le régime qu'ils avaient eux-mêmes subi avant 1950. Dans un tel contexte, face aux crises politiques et aux violations des droits fondamentaux, la revendication constitutionnaliste retrouva l'aspect symbolique et militant qu'elle avait déjà pu avoir au cours de l'histoire turque (notamment dans la période qui avait précédé la révolution "Jeunes-Turcs ») auprès de certains intellectuels, universitaires et officiers. Ces gens se mirent à penser que la démocratie parlementaire fonctionnait mal en Turquie parce que la Constitution, inadaptée, préservait mal l'existence libre des partis politiques, la liberté de la presse, l'autonomie des universités ou l'indépendance de la magistrature et que, par ailleurs, la dévolution exclusive de la souveraineté nationale à la Grande Assemblée qu'elle réalisait conduisait en réalité à une dictature de la majorité parlementaire et, donc, de son gouvernement.

$\mathrm{Au}$ cours du premier semestre 1960, la situation politique se détériora sous l'effet conjugué d'une agitation dans les universités et d'une crise due à une politique économique peu cohérente. De façon significative, les manifestations étudiantes, qui devaient précéder l'intervention militaire du 27 mai 1960, commencèrent après qu'un professeur de droit constitutionnel de l'Université d'Istanbul décida de refuser de faire son cours, une telle matière ayant cessé, selon lui, d'exister en Turquie.

\section{Les contradictions du processus de constitutionnalisation et le cercle vicieux de la démocratisation dans la Turquie contemporaine}

Le coup d'État du 27 mai 1960 devait contribuer à mettre à jour les contradictions fondamentales du processus constitutionnel et démocratique en Turquie. On sait, en effet, que cette intervention militaire s'afficha à l'époque comme un coup d'État progressiste, le préambule de la Constitution de 1961 allant même jusqu'à parler de «révolution». Il est vrai qu'elle déboucha sur l'un des régimes politiques les plus libéraux que la Turquie ait jamais connu. Une effervescence intellectuelle réformatrice, qui marqua toute une génération de professeurs de droit constitutionnel, accompagna l'élaboration d'une nouvelle constitution. On procéda à une analyse encyclopédique des constitutions du monde, pour donner aux rédacteurs du nouveau texte la meilleure actualité constitutionnelle. On n'hésita pas à innover dans de nombreux domaines, notamment dans celui de l'État de droit, en créant une cour constitutionnelle et en instaurant un contrôle de constitutionnalité, à une époque où de tels mécanismes n'étaient pas aussi courants qu'aujourd'hui. En outre, pour la première fois dans l'histoire turque, l'élaboration d'une constitution fut placée sous le contrôle d'une assemblée constituante composée de membres élus par les partis politiques et les 
organisations sociales. Le texte final fut soumis à référendum et son approbation ne fut pas que de pure forme, puisque plus de $38 \%$ de l'électorat turc refusa de ratifier le texte. Sa promulgation permit finalement la tenue, dès le mois d'octobre 1961, d'élections législatives qui confirmèrent, en dépit de la victoire des "Républicains ", l'existence d'une opposition active, fidèle aux « Démocrates » et désormais rassemblée autour du Parti de la justice de Süleyman Demirel.

Par la Constitution de 1961, la Turquie du début des années soixante se donnait néanmoins l'apparence d'une république audacieuse, qui corrigeait les imperfections de son ouverture des années cinquante et franchissait une nouvelle étape de sa modernisation politique en constitutionnalisant sa démocratie parlementaire (Kili S., 1971). Au moment où bon nombre de pays méditerranéens (l'Espagne, le Portugal, la Grèce) vivaient encore sous la dictature, la Turquie, qui par ailleurs avait refusé de s'associer dans les années cinquante au mouvement des non-alignés, apparaissait ainsi comme le bon élève de la "démocratie à l'occidentale » en Méditerranée orientale. Mais cette apparente euphorie politique ne pouvait faire oublier que, pour la première fois dans l'histoire de la République, l'armée était sortie de ses casernes, prenant par là même conscience du rôle qu'elle pouvait jouer dans le système politique turc. L'armée eut d'ailleurs le souci d'assurer, d'emblée, la pérennité de sa présence politique, en faisant élire par le Parlement à la présidence de la République le général Gürsel qui était à la tête du comité qui avait dirigé le coup d'État. En outre, cette normalisation politique s'accompagna d'une répression spectaculaire débouchant, notamment, sur le procès solennel des dirigeants démocrates et l'exécution de certains d'entre eux, dont le Premier ministre déchu, Adnan Menderes. Les anciens dirigeants démocrates étant principalement accusés, en l'occurrence, d'avoir voulu « changer, modifier et abroger de force la Constitution ", le constitutionnalisme fut là encore en vue mais bien sûr pas pour la meilleure cause.

Le coup d'État du 27 mai et la période d'instabilité qui l'avait précédé confirmaient, en fait, le décalage entre les projets d'une élite réformatrice et l'aptitude de la société civile à assimiler des réformes initiées de façon volontariste, mais ils montraient surtout la complexité qui était celle d'un processus de modernisation qui avait plus d'un siècle d'existence en Turquie. Car, désormais, ce processus devait être constitutionnel, démocratique et recevoir l'aval de la société civile. Dès lors, loin de déboucher sur une ère de sérénité politique, le coup d'État de 1960 allait conduire à de nouvelles phases d'instabilité.

À l'échec de la démocratisation des années cinquante, les constituants de 1961, qui incriminaient surtout la Constitution de 1924, avaient réagi par un texte ambitieux qui cherchait non seulement à instaurer des garanties contre les risques d'excès des gouvernants, en créant des contre-pouvoirs, mais aussi à émanciper la société civile par la reconnaissance de nouveaux droits et de nouvelles libertés. Fortement influencé par le constitutionnalisme de l'après-guerre (allemand et italien notamment), il se caractérisa ainsi par la mise en place d'un parlement bicaméral, la création d'une cour constitutionnelle, la reconnaissance solennelle de libertés et de droits individuels et sociaux. Il s'agissait, en fait, de franchir une nouvelle étape modernisatrice, en construisant un régime démocratique qui engendrerait une société nouvelle.

Toutefois, la mise en œuvre de cette " constitution d'architectes ", pour reprendre une expression du Pr. Erdogan Teziçilllustrant les velléités réformatrices de ce texte, allait conduire à d'autres périodes d'incertitude et à de nouvelles interventions de l'armée 
« coup de palais » de 1971 et coup d'État de 1980). Ces développements ont, bien sûr, mis en lumière toute la difficulté du passage à la démocratie en Turquie. Dans les autres pays méditerranéens, le processus de démocratisation, bien que parfois plus tardif, a été assez linéaire. Des phénomènes de transition, ayant d'ailleurs chacun leur spécificité, ont initié une mutation profonde des pratiques politiques et sociales, amenant à une constitutionnalisation réelle et durable du pouvoir. En Turquie, depuis la Seconde Guerre mondiale, on a assisté à des cycles d'ouverture, qui ont débouché sur des crises politiques et sociales sanctionnées par des interventions militaires.

Certes, l'objectif d'établir un régime démocratique constitutionnalisé n'a jamais été abandonné, comme si la Turquie était incapable d'envisager, pour elle-même, un autre type de régime, mais ce phénomène de cercle vicieux a entamé la crédibilité du processus de démocratisation dans ce pays. En premier lieu, il a favorisé des interventions militaires, qui ont mis en cause le fonctionnement normal des pouvoirs publics et se sont accompagnées de phases de répression sévère. Sans revenir sur les conséquences des coups d'État de 1960 ou de 1982, que nous avons précédemment évoquées, ou dont nous reparlerons, rappelons que le simple « coup de palais » de 1971 se solda par la mise en place d'un gouvernement de techniciens, l'interdiction de plusieurs partis politiques, vingt-neuf mois de loi martiale et une révision constitutionnelle restreignant plusieurs libertés publiques importantes. En deuxième lieu, ce cercle vicieux a montré l'immaturité de la société politique et les faiblesses de la société civile dans le processus de démocratisation. À la fin des années soixante-dix, notamment, tandis que le fonctionnement des institutions politiques parlementaires connaissait de réels problèmes (incohérence et instabilité des coalitions parlementaires), la Turquie sombra parallèlement dans une guerre civile larvée (assassinats politiques, affrontements dans les universités, activisme de partis extrémistes...). En dernier lieu, ce cercle vicieux a abouti à une implantation durable et inédite de l'armée dans un système constitutionnalisé. Cette implantation s'est opérée essentiellement par l'instauration, au sein même de l'ordre constitutionnel turc, d'un Conseil national de sécurité (cNs) représentant la plupart des instances militaires importantes. L'article 118 de la Constitution de 1982, dans sa version initiale, précisait, notamment, que

les décisions relatives aux mesures qu'il estime indispensables en vue de sauvegarder l'existence et l'indépendance de l'État, l'intégrité et l'indivisibilité du territoire ainsi que la paix et la sécurité de la société, sont prises en considération par le Conseil des ministres de façon prioritaire.

Cette rédaction, floue quant aux limites des compétences de cette institution, était en fait très révélatrice (Tanör, 1988). Ainsi, allait-on pouvoir saisir, très concrètement, l'influence déterminante qui pouvait être celle du CNS pendant l'expérience du gouvernement Erbakan en 1996/97. Les remontrances extrêmement vives et ciblées qu'il a alors formulées au chef islamiste du gouvernement en question - sur les orientations de sa politique - ont abouti à la démission de ce dernier, consacrant un processus de coup d'État en douceur que les intellectuels turcs ont qualifié, non sans humour, de « coup d'État post-moderne ».

\section{L'état du constitutionnalisme turc contemporain}

41 Les réformes institutionnelles réalisées par la Constitution de 1982 (retour au monocaméralisme, réformes de l'investiture des gouvernements, renforcement des 
pouvoirs du président de la République) ne sont pas parvenues à assurer une stabilité gouvernementale suffisante, notamment au cours de la décennie qui vient de s'écouler. Cette carence tient moins aux mécanismes constitutionnels actuels qu'au mode de scrutin et aux partis politiques. En dépit de son souci initial de rationalisation, la proportionnelle départementale (assortie d'une barre de représentativité minimum de $10 \%)$ employée pour les législatives n'a pas réussi à empêcher la multiplication des partis représentés au Parlement. Ce phénomène a encouragé une atomisation des forces politiques turques, qui a débouché, dans les années quatre-vingt dix, sur un recours systématique à des gouvernements de coalition, sans grande cohérence et sans grand avenir. Faut-il, pour autant, souhaiter que la Turquie s'oriente vers la mise en place d'un régime présidentiel ou semi-présidentiel ? Rien n'est moins sûr. D'une part, une telle option cadrerait mal avec la tradition constitutionnelle et politique turque qui, depuis 1946, a fait prévaloir un parlementarisme de type moniste. D'autre part, dans un pays où la pratique démocratique reste fragile malgré son antériorité, elle présenterait des risques non négligeables d'autoritarisme et de pouvoir personnel. La solution aux problèmes de stabilité du système politique turc est sans doute en l'occurrence moins institutionnelle que profondément politique (évolution des forces politiques vers plus de cohérence et de transparence) et sociale (poursuite des mutations de la société civile). La large victoire de l'AKP lors des élections législatives de novembre 2002, qui a débouché sur un parlement bipartisan (АКР/снР), a en outre relativisé l'urgence de ce problème (Marcou, 2003). En réalité, le débat constitutionnel de fond, qui s'est engagé dans le cadre des réformes destinées à permettre à la Turquie de satisfaire aux critères de Copenhague, concerne, pour l'essentiel, la place de l'armée et les libertés fondamentales. Il s'agit en fait de sortir ce pays d'un "constitutionnalisme sécuritaire » où, malgré la présence d'un appareillage institutionnel libéral, l'armée tient un rôle de gendarme du système et où les libertés fondamentales se voient malgré tout limitées dans une partie de leur garantie.

42 Depuis que le sommet européen d'Helsinki a reconnu, en 1999, la vocation de la Turquie à être candidate à l'UE, des réformes constitutionnelles et législatives spectaculaires ont été conduites par le gouvernement de Bülent Ecevit (1999-2001) et par les gouvernements Gül et Erdogan de l'AKP, issus des élections législatives de novembre 2002.

43 Ces réformes ont d'abord concerné le cNs et cherché, plus généralement, à réduire le rôle politique de l'armée (Dorronsoro, 2004). La très importante révision du 17 octobre 2001 a fait du CNS une institution principalement composée de civils et ne lui reconnaît plus, en réalité, qu'un rôle consultatif. Une nouvelle révision, adoptée en mai 2004, s'est attachée à réduire encore l'influence de l'armée dans le fonctionnement des pouvoirs publics (fin de la présence militaire au sein du Haut Conseil de l'éducation - Yök abrogation de la justice d'exception, notamment des tribunaux de sûreté de l'État, instauration d'une transparence du contrôle des biens publics de l'État détenus par l'armée...). Mais depuis la victoire de l'AKP, la révision de la Constitution fait l'objet d'une rivalité sourde entre «l'État profond» et le gouvernement de Recep Tayyip Erdogan. La place de l'armée dans le système politique turc est ainsi devenue l'enjeu majeur du processus de réformes entrepris en Turquie pour satisfaire aux critères de Copenhague. Les révisions de 2001 et de 2004 ainsi que les réformes législatives afférentes ont formellement entamé le rôle du cNs, mais ce n'est que par une pratique régulière et par le développement d'une culture démocratique au sein même de l'État 
que le déclin de cette institution anachronique sera définitivement confirmé et que l'armée trouvera une place véritablement conforme à celle qui doit être la sienne dans un système constitutionnalisé.

Ces réformes ont aussi largement touché la deuxième partie de la Constitution de 1982, consacrée "aux droits et devoirs fondamentaux » (Kaboglu, 2004). Les lacunes dans la garantie des libertés alimentaient, en effet, une critique extrêmement vive contre ce texte constitutionnel, tant à l'intérieur du pays que sur la scène européenne. La révision du 17 octobre 2001 a constitué là encore un pas essentiel, dotant la Turquie d'un système de protection des droits, désormais très proche des standards européens. L'une des mesures les plus importantes en la matière a été la remise en cause du double régime de limitation aux droits et libertés, établi par l'art. 13 dans sa rédaction initiale. Au-delà des limitations spécifiques constituant le régime d'organisation et de réglementation des libertés publiques - que l'on retrouve dans tous les États constitutionnalisés - le système turc érigeait une faculté complémentaire de limitation générale qui pouvait permettre d'anéantir facilement les libertés considérées comme les plus dangereuses par le pouvoir. Désormais, les droits et libertés fondamentaux ne peuvent être limités que pour des motifs prévus par des dispositions particulières de la Constitution et en vertu de la loi, et pour autant que ces limitations ne portent pas atteinte à l'essence même des droits et libertés. En outre, les limitations dont les droits et libertés fondamentaux font l'objet ne peuvent être en contradiction ni avec la lettre et l'esprit de la Constitution ni avec les exigences d'un ordre social démocratique et laïque et doivent respecter le principe de proportionnalité.

45 La négociation et la conclusion de l'Accord d'union douanière avec l'Union européenne avaient donné lieu à une première réforme constitutionnelle d'envergure (suppression des références au coup d'État dans le préambule, abaissement de la majorité électorale à 18 ans, possibilité de voter pour les Turcs résidant à l'étranger, amélioration du statut des syndicats et des partis). Les révisions de 2001 (qui ont touché plus du cinquième des 177 articles de la Constitution) et de 2004 (qui touchent des sujets très symboliques comme la peine de mort, la justice d'exception ou l'égalité entre homme et femme) se sont, en outre, accompagnées de près d'une dizaine de "paquets d'harmonisation législative». Il s'agit de trains de réformes législatives thématiques destinés à accompagner, en profondeur, l'effort constitutionnaliste réalisé par des mesures concrètes, touchant les secteurs les plus cruciaux dans le développement d'un État de droit (police, justice, statut de la personne, procédure pénale...). Ainsi l'ampleur des réformes, conduites dans la perspective de l'intégration européenne, est en train de transformer profondément le système sécuritaire, hérité du coup d'État de 1980 (Dorronsoro, 2004). Dans cet ordre d'idée, on peut s'interroger, comme l'avait fait la conférence internationale de droit constitutionnel d'Ankara - organisée en janvier 2001 par les avocats turcs - sur le devenir de la Constitution de 1982 et sur sa capacité à incarner la nouvelle donne d'un constitutionnalisme émancipateur, plus conforme aux traditions européennes et à l'état de développement de la société civile turque contemporaine.

Toutefois, il faut bien voir que dans le contexte actuel du gouvernement post-islamisme de l'AKP suspecté par certains de vouloir remettre en cause la république laïque, l'idée d'une refondation constitutionnelle est devenue un sujet extrêmement sensible. Dès lors, c'est avant tout le pragmatisme qui paraît prévaloir, quitte à ce que la nature profonde de la Constitution de 1982 en soit profondément transformée. Ainsi, il n'est 
pas impossible que le texte constitutionnel, à l'origine le plus terne qu'ait connu la Turquie, préside à l'achèvement de la constitutionnalisation du pouvoir dans ce pays. Il y aurait là une revanche du constitutionnalisme, sans doute méritée, sur une histoire tumultueuse.

\section{BIBLIOGRAPHIE}

BAdIE B., 1986, Les Deux États, Fayard, Paris.

Dorronsoro G., 2004, « La Turquie, une démocratie sous contrôle » dans Olivier Roy (dir.), La Turquie aujourd'hui, Paris, Éd. Universalis.

Dumont P., 1989, « La période des Tanzimat (1839-1878) », dans Mantran R. (dir.), Histoire de l'Empire ottoman, Paris, Fayard, 1989.

FESH P., 1909, Les Jeunes Turcs, Paris, Paul Paclot Librairie-Éditeur.

FuAd BAsGil A., 1939, La Turquie, Constitution et régime politique, Paris, Delagrave, « La vie juridique des peuples ».

Kaboglu I. O., 2004, «Vers le droit constitutionnel des libertés en Turquie » dans Essays in Honour of Georgios I. Kassimatis, Bruxelles, Bruylant.

KIII S., 1971, Turkish constitutional developments and Assembly debates on the Constitutions of 1924 and 1961, Istanbul, Robert College Research Center Yayinlari.

LEWIs B., 1988, Islam et Laïcité, Paris, Fayard.

LeWIs B., 1993, Le Retour de l'Islam, Paris, Gallimard, « Folio ».

Marcou J., 2000, « La laïcité en Turquie, une vieille idée moderne », Confluences Méditerranée, avril 2000.

- 2003, «La victoire de l'AKP aux élections législatives de novembre 2002 en Turquie » (en arabe) dans la revue égyptienne Al Siayassa Al Dawliya (Politique internationale), $\mathrm{N}^{\circ} 151$, janvier.

Marcou J, Üstel F., VARDAR D., 2000, « La République en France et en Turquie », Revue Internationale de Politique Comparée, vol.7, $\mathrm{n}^{\circ}$ 3, p. 543.

UlusLARARAsi ANAYASA HuKUKu KuRULTAYI, 2001, Actes de la Conférence Internationale de droit constitutionnel, organisée par l'Union des Barreaux d'avocats de Turquie, 9-13 janvier 2001, Ankara, Tûrkiye Barolar Birligi Yayinlari, (en turc).

\section{NOTES}

1. Entretien avec le Professeur Erdogan Teziç, recteur de l'Université de Galatasaray, devenu président du Yök en 2003 (décembre 1994, janvier 2001). 
INDEX

Mots-clés : histoire constitutionnelle, Turquie, Constitution

\section{AUTEUR}

JEAN MARCOU

Filière francophone d'économie et de sciences politiques de l'Université du Caire / IEP Grenoble 